\title{
Historicidad del concepto "salud del trabajador" en el ámbito de la salud colectiva: el caso de Brasil
}

\author{
The history of the concept of "worker health" in the field of \\ collective health: the case of Brazil
}

\section{INTRODUCCIÓN}

Actualmente, los problemas que afectan la salud y la calidad de vida de la clase trabajadora están adquiriendo una mayor visibilidad social. En el ámbito académico ha surgido una variedad de estudios y prácticas de indiscutible valor, incluso en la ausencia de una adecuada precisión conceptual sobre el carácter de la asociación entre el trabajo y el proceso salud-enfermedad. En este editorial se presenta una discusión sobre las concepciones que han pautado los diversos actores que abordan esa cuestión, sea en el desarrollo de estudios e investigaciones o en el direccionamiento de las acciones prácticas. Esta reflexión tiene como base la experiencia brasileña en este tema.

La relación entre el trabajo y el proceso salud-enfermedad, aunque constatada desde la antigüedad, pasó a constituirse en foco de atención a partir de la Revolución Industrial. Las propuestas de intervención en las empresas se concretaron en una sucesión de normalizaciones y legislaciones que tuvieron su punto más relevante en Inglaterra con el Factory Act de 1833. En la segunda mitad del siglo XIX surgió la medicina del trabajo, dirigida hacia el espacio restricto de la fábrica, centrada en la figura del médico bajo una visión eminentemente biologicista e individual y bajo una interpretación unívoca y unicausal de los agravios. Ese planteamiento podría ser resumido en la idea de que para cada enfermedad habría un agente etiológico.

Un avance conceptual y operacional con relación a la medicina del trabajo llegó con el concepto de salud ocupacional, una propuesta interdisciplinar y multicausal que viene desarrollándose desde las primeras décadas del siglo pasado y se funda en la idea de higiene industrial, que entiende la ocurrencia de la enfermedad en el trabajo como consecuencia de la exposición a un conjunto de factores de riesgo -físicos, químicos, biológicos y mecánicos- presentes en el ambiente laboral. Ese concepto se basa en el modelo de la historia natural de la enfermedad (1), que deriva de la interacción constante entre el agente, el hospedero y el ambiente, sin contextualizar las razones del origen de esa relación.

En Brasil, a medida que históricamente se construía el concepto en la salud colectiva, se delineaba en paralelo el marco conceptual y de prácticas que hoy se configura como el campo de la salud del trabajador, cuyo objeto, de forma amplia, es el proceso de salud-enfermedad de los grupos humanos en su relación con el trabajo, objeto de interés tanto del sector salud como de los trabajadores y empresarios (2).

La concepción salud del trabajador tiene raíces en la corriente latinoamericana de la medicina social y, más específicamente, en el movimiento por la Reforma Sanitaria brasileña, inspirado en varios movimientos mundiales comprometidos con la universalización y la promoción de la salud. En Brasil, esa ola reformista en el sector salud coincidió con una coyuntura política de organización y de luchas por la redemocratización del país -sobre todo a partir de la década de 1980- en la que varios 
actores, situados en espacios y lugares sociales diferentes, confluyeron en sus cuestionamientos al modelo hospitalocéntrico de las políticas públicas del sector. Teniendo como premisas las cuestiones sociales que afectan la salud, el movimiento sanitario centró su análisis en el papel del trabajo en la reproducción social de las poblaciones. El avance de la producción académica en medicina preventiva y social y en salud pública permitió ampliar el cuadro interpretativo de los procesos de trabajo dentro de las dimensiones de clase y sus aspectos culturales de sumisión y resistencia, y en su asociación con la salud y la posibilidad de enfermedad de los trabajadores y de sus familias.

En el campo de la salud del trabajador, teóricamente, los trabajadores se constituyen en sujetos políticos colectivos, depositarios de un saber emanado de la experiencia y deben ser considerados agentes esenciales de acciones transformadoras. La incorporación de ese saber es decisiva, tanto en el ámbito de la producción de conocimientos como en el desarrollo de las prácticas de atención a la salud. El reconocimiento de ese saber/poder ha sido el pilar del "Modelo Obrero Italiano" (3), que emergió del dinamismo de los movimientos sociales, a fines de la década de 1970, teniendo como foco particular el cambio y el control de las condiciones de trabajo en las unidades productivas. La no-delegación -expresada en el rechazo a transferir a los técnicos o representantes sindicales la tarea de sistematizar el conocimiento obtenido por los grupos sometidos a las mismas condiciones de trabajo (grupos homogéneos)- y la validación consensual -resultante de la discusión colectiva de las evaluaciones que pautarían los procesos reivindicatorios- se constituyeron en los presupuestos básicos de ese modelo.

Así, la producción de conocimientos en el campo de la salud del trabajador, en el ámbito de la salud colectiva, tiene como marco definidor la comprensión de los diversos niveles de complejidad de las relaciones entre el trabajo y la salud, incluyéndose ahí la visión y la participación de los trabajadores y, como concepto nucleador, el de proceso de trabajo (4-6). Este último, extraído de la economía política (7) en su acepción marxista, es entendido como el escenario primario de la explotación y de la confrontación de clase, si es adoptado en toda su extensión teórica, y tiene un elevado poder explicativo de la génesis de los agravios a la salud en los diferentes colectivos de trabajadores.

De esta forma, el concepto más universalmente conocido de salud ocupacional se diferencia de la noción de salud del trabajador. El primero, desde un punto de vista disciplinar y profesional es menos complejo: abarca fundamentalmente las áreas de medicina y de ingeniería de seguridad. El segundo se centra en el estudio del proceso de trabajo desde el punto de vista de las ciencias sociales, la epidemiología y la planificación, a las que se suman, de ser necesario, nociones de demografía, estadística, ecología, geografía, antropología, economía, sociología, historia, ciencias políticas, toxicología, ingeniería de producción y ergonomía.

Resulta necesario señalar algunos puntos respecto de los estudios que toman como marco los procesos de trabajo. En primer lugar, es importante decir que tales estudios exigen un tratamiento interdisciplinario. Esta visión tiene como premisa la substitución del "principio de jerarquía" entre los saberes por el "principio de cooperación" y supone el diálogo, la interacción y el cuestionamiento recíproco, aproximándose a la filosofía y a la acción comunicativa (8). Dentro de tales principios, se deben tener en cuenta al menos dos planos: uno que contempla el análisis del contexto histórico, social, económico, político y cultural de las relaciones sociales de producción, de los espacios de trabajo y de las condiciones de reproducción de los trabajadores, y otro que se refiere a determinadas características técnicas de los procesos de trabajo que puedan potencialmente repercutir en la salud y en la subjetividad de los trabajadores (6). Para analizar estos dos planos existen algunas nociones y algunos conceptos mediadores, entre los cuales, los más comúnmente utilizados son riesgo, carga de trabajo, exigencias o solicitudes. Estos tienen relación con condiciones materiales, dimensiones epidemiológicas y componentes cualitativos derivados de la organización del trabajo y deben ser usados para identificar y analizar situaciones generadoras de efectos potenciales o reales sobre la salud de grupos, categorías o sectores.

Un segundo aspecto a resaltar es que, a pesar de que en teoría se sepa que el análisis de los efectos de los procesos de trabajo sobre la salud requiere la conformación de equipos de investigadores de diversas áreas, eso raramente ocurre en la práctica. Una de las limitaciones de la producción científica se relaciona con el desfasaje entre el incuestionable avance desde el punto de vista teórico para 
aprehender la complejidad de las cuestiones relativas a la salud de los trabajadores y el nivel de los resultados empíricos alcanzados en los estudios. Esa ausencia de colaboración interdisciplinaria termina produciendo un conocimiento reduccionista que contribuye a comprender una dimensión de los problemas, pero oculta o desconoce otras tanto o más importantes. Esto ocurre, sobre todo, con los análisis epidemiológicos que se apartan intencionalmente de los aspectos sociales y políticos implicados en los problemas de salud derivados del trabajo. Resulta necesario reiterar que ninguna disciplina aislada consigue abordar la amplitud de la relación proceso trabajo-salud en sus múltiples e imbricadas dimensiones. Sin embargo, es preciso evitar la incorporación, sin el debido rigor, de los conceptos de otras disciplinas que, por su falsa apariencia de transitividad, esconden profundas diferencias y llevan a análisis fragmentados y a síntesis simplificadoras.

Un tercer punto que necesita quedar claro es que el concepto de proceso de trabajo surgió asociado a la conformación del proletariado industrial urbano que, en los últimos años, ha sufrido profundas transformaciones derivadas de una nueva lógica productiva que redunda en cambios en la composición de la fuerza de trabajo, en la introducción de nuevos patrones de tercerización, subcontratación y precarización laboral. Hoy el mundo laboral es mucho más complejo y variado. Por ejemplo, los estudios de los sectores de servicios constituyen un desafío para los que se acostumbraron a trabajar con el concepto de proceso de trabajo, cuando lo que está en juego son formas de "trabajo en proceso". Aunque en esas áreas existan algunas características análogas al trabajo industrial, hay atributos de alta significancia y especificidad en la interacción entre trabajadores y clientes/usuarios/destinatarios/consumidores. Asimismo, caben adecuaciones al estudio de segmentos no directamente determinados por la ley del valor o que no viven del trabajo asalariado. De la misma forma, algunas cuestiones antes no consideradas pasaron a merecer la atención de los profesionales que tratan con los trabajadores, como es el caso de las relaciones entre salud mental y trabajo. Hoy, nociones como acoso moral, sufrimiento y estrés afloraron como problemas en los más diversos sectores de actividad, y sobre las que existe muy poco consenso. En consecuencia, además de la necesidad de que sean efectuados ajustes y adaptaciones en la aplicación del concepto de "proceso de trabajo", los estudiosos enfrentan el desafío de encontrar categorías y conceptos adecuados para comprender la multiplicidad de nuevas inserciones de los trabajadores en el mundo productivo.

Finalmente, al focalizar el campo de salud del trabajador no se puede perder de vista la estrecha interrelación entre la producción, el consumo, el uso de recursos naturales y el impacto que esas acciones humanas provocan en el medio ambiente y en la población.

\section{BAJO EL ÁNGULO DE LA SALUD PÚBLICA}

Inicialmente, cabe mencionar algunos antecedentes que influenciaron la institucionalización del área de la salud del trabajador en el sistema público de salud. En 1983, la Organización Panamericana de la Salud (OPS), divulgó el documento "Programa de acción en la salud de los trabajadores" con directrices para la implantación de programas en la red pública de servicios de salud. En esa misma línea, la Organización Internacional del Trabajo (OIT), en 1985, acompañando una tendencia internacional de ampliación de derechos de los trabajadores, adoptó la "Recomendación sobre los servicios de salud en el trabajo", cuyas principales características son el principio de la amplia participación de los trabajadores, la actuación en equipos multiprofesionales y su implementación, fundamentalmente, a partir de políticas públicas.

En esa dirección, el fortalecimiento del movimiento de los trabajadores, por la conquista de derechos elementales de ciudadanía y por la consolidación del derecho a la libre organización, condujo a una ampliación de las reivindicaciones laborales, introduciendo en ellas cuestiones relacionadas a la salud y, más aun, demandando servicios de atención en la red pública. En respuesta a esa demanda, en Brasil se crearon programas y centros de referencia en salud del trabajador, con diversos grados de 
participación de sus representantes en la formulación y en el desarrollo de las acciones. Un paso significativo, a nivel nacional, fue la redacción de la Constitución Federal de 1988, que Ilegó precedida por la realización de la VIII Conferencia Nacional de Salud, en 1986, con amplia participación social, y de la I Conferencia Nacional de Salud de los Trabajadores, en ese mismo año. Tales eventos, que constituyen marcos históricos en la lucha por la defensa de la salud como derecho y por el Sistema Único de Salud (SUS), tuvieron como desdoblamientos la promulgación de la Ley Orgánica de Salud, las leyes orgánicas municipales y los códigos de salud, en un movimiento de profundización y de interiorización de los derechos por medio de la municipalización de los servicios de atención.

Desde la I Conferencia Nacional de Salud de los Trabajadores, las entidades representativas de los trabajadores y de la sociedad civil, así como los órganos gubernamentales y técnicos, han propuesto la formulación de una Política Nacional de Salud de los Trabajadores en la esfera de la salud pública. Una política basada en los principios del SUS, o sea, que garantice el acceso universal e integral, con énfasis en acciones de prevención y de promoción, descentralización y participación social. Bajo tales premisas fue establecida la Red Nacional de Atención Integral a la Salud del Trabajador (RENAST), en 2002 (9). Cabe a la RENAST promover acciones asistenciales, de vigilancia y de promoción de la salud en la red SUS, articulando líneas de cuidado en la atención básica, ambulatoria, prehospitalaria y hospitalaria de media y alta complejidad, bajo el control social, en los tres niveles de gestión: nacional, de los estados y de los municipios. Esa red está compuesta por Centros de Referencia en Salud del Trabajador (CEREST) que asumen el papel de soporte técnico-científico y de articulación y acuerdo de las acciones de salud, intra e intersectorialmente, en su territorio de alcance. Su misión en el SUS es, pues, ser un polo irradiador de una cultura de protección de los trabajadores y de prevención de las enfermedades del trabajo, donde estén localizados: en la capital, en las áreas metropolitanas y en los municipios polos de asistencia de las regiones y microrregiones de salud.

Por último, en 2012, fue instituida oficialmente la Política Nacional de Salud del Trabajador y de la Trabajadora (10), la cual refleja de forma pormenorizada el reconocimiento del patrimonio acumulado a lo largo de las últimas décadas por sectores académicos, movimientos de trabajadores y profesionales que actúan en los servicios. En ella están definidos los principios, las directrices y las estrategias a ser observados por las tres esferas de gestión del SUS para el desarrollo de la atención integral a la salud de el/la trabajador/a, objetivando la promoción y la protección de su salud y la reducción de la morbimortalidad derivados de la enfermedad y de los agravios en las actividades laborales. Esa política se integra al conjunto de políticas de salud del SUS, que contemplan la transversalidad de las acciones de salud y el trabajo como uno de los determinantes del proceso salud-enfermedad. Son sujetos de esa política:

\footnotetext{
...todos los trabajadores, hombres y mujeres, independientemente de su localización, urbana o rural, de su forma de inserción en el mercado de trabajo, formal o informal, de su vínculo laboral, público o privado, asalariado, autónomo, cuentapropista, temporario, cooperativizado, aprendiz, pasante, doméstico, jubilado o desempleado. (10 Art. 3) (Traducción nuestra)
}

Uno de los objetivos prioritarios de la Política Nacional de Salud del Trabajador y de la Trabajadora es el fortalecimiento de la Vigilancia en Salud del Trabajador (VISAT), cuyo objeto central de intervención es el proceso de trabajo y su relación con la salud, teniendo como objetivo controlar riesgos y disminuir accidentes y enfermedades (11). El proceso de vigilancia parte de la situación de riesgo o del agravio, incluso de la conjunción de ambas situaciones, y se fortalece cuando está integrado a la noción de territorio, en el que diversos actores e instancias institucionales desempeñan sus acciones, configurando un campo de fuerzas en torno al proceso de trabajo, representado por la actividad y por el sector donde se concentran los casos y las exposiciones. La vigilancia, por lo tanto, es comprendida, en el ámbito de la política, como un medio de fortalecimiento de los trabajadores en la lucha por la salud y como una práctica compleja, amplia, permeada por múltiples intereses algunas veces conflictivos, y no meramente como una práctica neutra, estandarizada y estrictamente técnica $(12,13)$. 
La Política Nacional de Salud del Trabajador y de la Trabajadora elaboró directrices y estrategias para la implementación de las acciones de vigilancia en salud:

a. identificación de las actividades productivas de la población trabajadora, de las situaciones de riesgo y de las necesidades, demandas y problemas de salud de los trabajadores en el territorio;

b. intervención en los procesos y ambientes de trabajo;

c. producción de tecnologías de intervención, de evaluación y de monitorización de las acciones de vigilancia;

d. control y evaluación de la calidad de los servicios y programas de salud del trabajador de las instituciones y empresas públicas y privadas;

e. producción de protocolos, de normas técnicas y reglamentos.

Entre las estrategias se encuentran las propuestas de:

a. Articulación intrainstitucional dentro de todas las instancias del SUS: atención básica (Programa de Salud Familiar, urgencia y emergencia), servicios de media y alta complejidad, instancias de vigilancia epidemiológica, sanitaria y ambiental y con proyectos relacionados, otras políticas de salud como las referentes al cáncer, a la violencia urbana y a la salud mental. Esa articulación intrainstitucional es una de las mayores trabas a ser superada en el área de la salud de los trabajadores.

b. Articulación intersectorial comprendida como el ejercicio de la transversalidad en las políticas de la Vigilancia en Salud del Trabajador junto con sectores del Ministerio de Trabajo, de Previsión Social, de Medio Ambiente y del Ministerio Público. Un progreso en esa dirección se produjo con la promulgación, en 2010, de la Política Nacional de Seguridad y Salud en el Trabajo (14), integrada por el Ministerio de Salud, el de Trabajo y el de Previsión Social.

c. La participación de los trabajadores o de sus representantes en la formulación, en el planeamiento, en el acompañamiento y en la evaluación de las acciones de vigilancia en salud.

Conviene recordar que el control social se concibe en la política pública brasileña como instrumento de vigilancia, de acción y de intervención por parte de los segmentos organizados de la sociedad civil sobre las acciones del Estado. Desde la promulgación de la Ley Orgánica de la Salud (15), el sector salud ha privilegiado, de forma pionera, la construcción democrática de las decisiones y ha atribuido a los representantes de la sociedad el derecho y el deber de fiscalizar y controlar a los gestores en las tres esferas de gobierno. Como espacios e instrumentos de esa participación se crearon los consejos y las conferencias de salud en las tres esferas de gobierno -federal, de los estados y de los municipios- como espacios estratégicos para la definición, elaboración, implementación y fiscalización de las políticas de salud. En este caso específico, fueron definidas por el SUS y vinculadas a los consejos de salud las Comisiones Intersectoriales de Salud del Trabajador, cuyas decisiones implican también enfrentar los conflictos propios de las relaciones sociales de trabajo y de las condiciones generales de reproducción de los trabajadores y de sus familias.

\section{AVANCES Y LAGUNAS}

En primer lugar, es preciso constatar que, en Brasil, la producción científica en este campo presenta una tendencia continua de crecimiento en las últimas décadas, universalizándose por muchas instituciones universitarias, abarcando diversas áreas del conocimiento y recibiendo incluso el influjo de las contribuciones de los profesionales que actúan en los servicios de salud. Ese incremento acompaña la multiplicación de los cursos de posgrado en el país, principalmente en el campo de la salud colectiva. De la misma forma que en otros ámbitos del conocimiento, el número mayor de investigaciones y textos publicados se concentra en universidades federales, de los estados y pontificias. 
Por otro lado, recientemente se establecieron grandes directrices de la Política Nacional de Salud del Trabajador y de la Trabajadora y los marcos regulatorios de sus atribuciones. No obstante, falta todavía mucho para aproximarse a las especificidades del mundo del trabajo que insisten en ser mucho más dinámicas que todas las teorías construidas para explicarlas o comprenderlas. Ciertamente, dada la complejidad de la actual coyuntura, cualquier análisis de los agravios a la salud de los trabajadores en Brasil, o en cualquier parte del mundo, será parcial e incompleta, no solo por lapsus intelectuales sino, sobre todo, porque en ese campo la búsqueda de soluciones frecuentemente se confronta con intereses económicos poderosos, arraigados e inmediatistas.

Finalmente, pensando desde una perspectiva latinoamericana es importante señalar como auspiciosa la medida de Salud Ambiental y del Trabajador del Mercosur (16), recientemente oficializada, y que tiene por objetivo armonizar los conceptos comunes a los Estados miembros. Esa medida fue acordada unánimemente entre los representantes de los países que integran la Comisión Intergubernamental de Salud Ambiental y del Trabajador, para facilitar una homogeneización de criterios en relación a la salud en los espacios laborales.

Minayo Gómez, Carlos

Investigador titular, Escola Nacional de Saúde Pública, Fundação Oswaldo Cruz, Brasil. minayogo@ensp.fiocruz.br

\section{REFERENCIAS BIBLIOGRÁFICAS}

1. Leavell H, Clark EG. Medicina Preventiva. São Paulo: McGraw-Hill; 1976.

2. Lacaz FAC. O campo Saúde do Trabalhador: resgatando conhecimentos e práticas sobre as relações trabalho-saúde. Cadernos de Saúde Pública. 2007;23(4):757-766.

3. Oddone I, Marri G, Gloria S, Briante G, Chiatella M, Re A. Ambiente de trabalho: a luta dos trabalhadores pela saúde. São Paulo: Hucitec; 1986.

4. Laurell AC, Noriega M. Processo de produção e saúde: trabalho e desgaste operário. São Paulo: Hucitec; 1989.

5. Mendes R, Dias EC. Da medicina do trabalho à saúde do trabalhador. Revista de Saúde Pública. 1991;25(5):341-349.

6. Minayo-Gómez C, Thedim-Costa SMF. A construção do campo da saúde do trabalhador: percurso e dilemas. Cadernos de Saúde Pública. 1997;13(Supl. 2):S21-S32.

7. Marx K. O Capital: Livro I, Capítulo VI (inédito). São Paulo: Ciências Humanas; 1978.
8. Habermas J. Teoría de la acción comunicativa. 2a ed. Madrid: Taurus; 1988.

9. Brasil, Ministério da Saúde. Portaria $N^{\circ}$ 1.679/GM [Internet]. 19 sep 2002 [citado 12 oct 2012]. Disponible en: http://www.saude.al.gov. br/sites/default/files/portaria_n.1679_de_18.09.2 002.pdf.

10. Brasil, Ministério de la Salud. Portaria $N^{\circ}$ 1.823 [Internet]. 23 ago 2012 [citado 12 oct 2012]. Disponible en: http://bvsms.saude.gov. br/bvs/saudelegis/gm/2012/prt1823_23_08_201 2.html.

11. Machado JMH. Alternativas e processos de Vigilância em Saúde do Trabalhador: a heterogeneidade da intervenção. [Tese de doutorado]. Rio de Janeiro: ENSP/Fiocruz; 1996.

12. Pinheiro TMM. A construção da vigilância em saúde do trabalhador no Sistema Único de Saúde (SUS): a vigilância do conflito e o conflito da vigilância. [Tese de doutorado]. Campinas: UNICAMP; 1996.

13. Baker EL. Sentinel Event Notification Systen for Occupational Risks (SENSOR): the concept. American Journal of Public Health. 1989;79 (Suppl):S18-S20. 
14. Brasil, Presidência da República. Decreto No 7.602 [Internet]. 7 nov 2011 [citado 12 oct 2012]. Disponible en: http://www.planalto.gov.br/ccivil_03/_Ato2011-2014/2011/Decreto/D7602.htm.

15. Brasil, Presidência da República. Lei $N^{0}$ 8.080 [Internet]. 19 set 1990 [citado 12 oct 2012]. Disponible en: http://www.planalto.gov. br/ccivil 03/leis/L8080.htm.
16. Argentina, Ministerio de Salud. Resolución 269/2012 [Internet]. 10 feb 2012 [citado 12 oct 2012]. Disponible en: http://www.infoleg. gov.ar/infolegInternet/anexos/190000194999/194280/norma.htm.

\section{FORMA DE CITAR}

Minayo Gómez C. Historicidad del concepto "salud del trabajador" en el ámbito de la salud colectiva: el caso de Brasil. [Editorial]. Salud Colectiva. 2012;8(3):221-227. 